\title{
The Analysis of Link Throughput of OFDM System in Broadband Satellite
}

\author{
Zhihui Li ${ }^{a}$, Qingyang Guan' ${ }^{b}$ Xiaoxu Liuc \\ School of Electronic and Information Engineering, Shenyang Aerospace University, Shenyang 100136, China \\ a411787748@qq.com, buan_qy@163.com, cxiaoxu955@163.com
}

Keywords: broadband satellite, link throughput, AMC, CQI

\begin{abstract}
In order to improve the link throughput of OFDM system in aviation broadband satellite, it is possible to combine the AMC to complete the modulation and coding of the signal. This paper established an AMC link model, including the movement model of aviation satellite, wave beam model of AMC, the way of code modulation to AMC, CQI and then analyzed the performance of link throughput according to the link model.
\end{abstract}

\section{Introduction}

At present, aims at the analysis of throughput for terrestrial wireless OFDM transmission systems, the analytical methods about link throughput are introduced detailedly in many literatures.[1-3]. In term of the aeronautical satellite broadband system, the system link focuses on the communication link in physical layer which is coveraged with the single wave speed within the aeronautical satellite and the user terminals. As for the analysis of the link throughput in aeronautical satellite broadband communication system, a link system within the coverage of the wave beam is neededto be established. And using the simulation to analyze the performance of the link throughput. The performance is related to many factors, such as: the atellite trajectory, the speed of movement, the system bandwidth, and so on. Combining with the AMC, the link throughput of OFDM System in aviation broadband satellite will be analyzed in this part.

\section{Analysis}

\subsection{The analysis process of the link throughput}

A link model of OFDM system is established in this section first.

The average throughput of users can be obtained by modeling a system link. For the link layer model of satellites, a beam coverage model needs to be established. Non-fixed area spot beam coverage is used in this paper. For aeronautical satellite communication systems using multiple beam antennas. Figure 1 is the analysis process. Figure 2 is the flow charts of the simulation of the link throughput. Initially, the parameters are initialized. And then establishing the communication link. According to the CQI, the satellite performs adaptive modulation and coding method selection. 


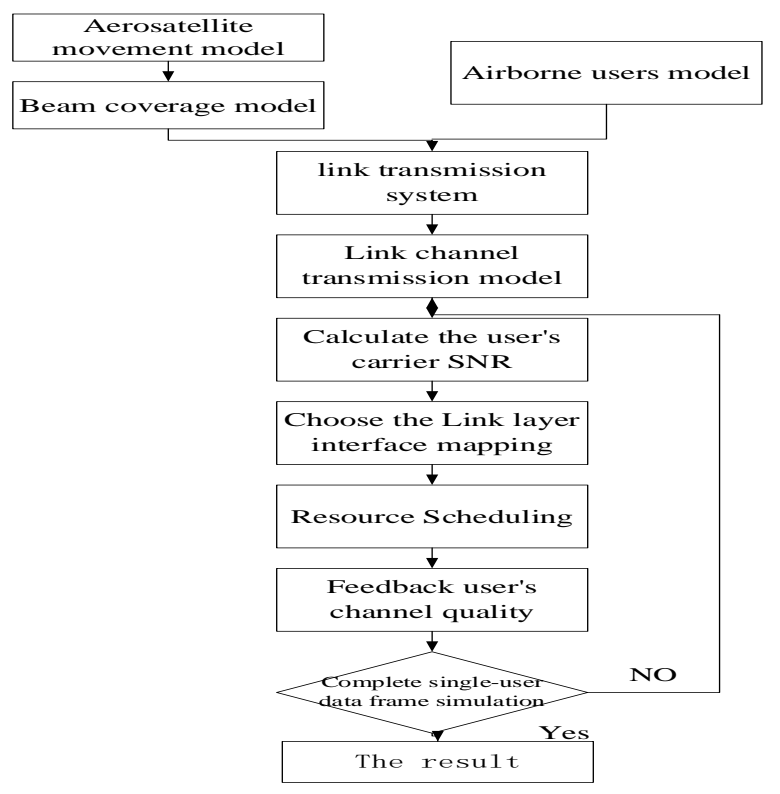

Fig. 1 The analysis process of OFDM system throughput

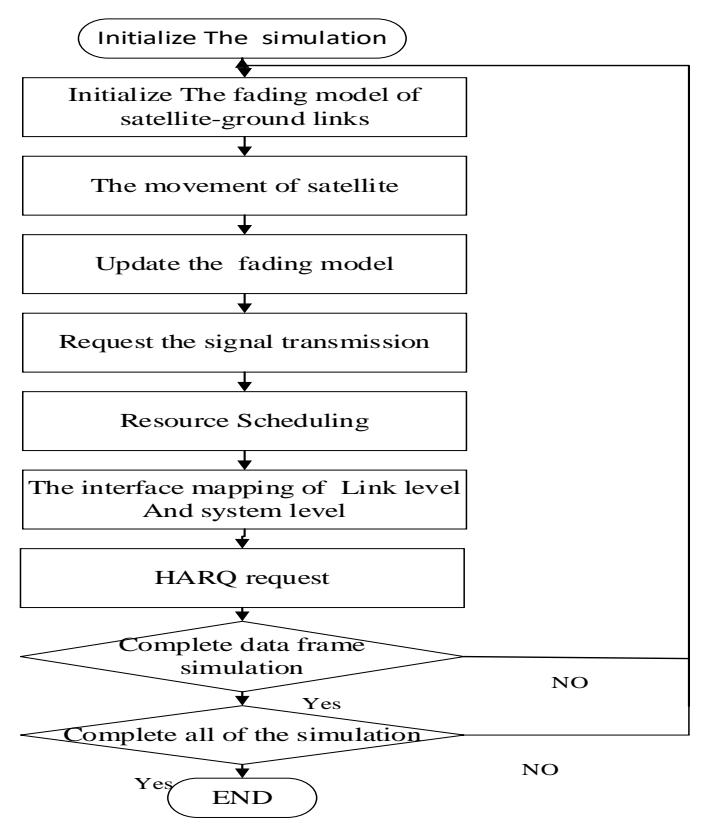

Fig. 2 The flow charts of the simulation of the link throughput

\subsection{The performance analysis of system link throughput}

An aviation satellite simulation model with the height of $770 \mathrm{~km}$ was established. Set the number of spot beams is 7 , the spot beam cover diameter is $450 \mathrm{~km}$, the satellite speed is $7.15 \mathrm{~km} / \mathrm{s}$, use $\mathrm{L}$ band as the carrier frequency, the simulation scenario selects multipath model parameters, and the channel model has direct The 3-path model of the main path component, the main path obeys the Rician distribution, and the maximum extension delay is 250ns. Take the maximum extended delay of the OFDM symbol cyclic prefix length greater than the multipath, the guard interval is 1us, and the symbol period of the OFDM can be taken as 1us $5=1$ us. Let 20 OFDM symbols constitute a subframe, and each 5 subframes constitute a radio frame, block-based pilot signals are used for channel estimation. Each basic simulation step is 1 subframe, the number of carriers is 1024, and the transceiver antenna model is SISO.Table 1 shows the parameter setting of OFDM system of aviation satellite. In order to simplify the simulation model, the aviation satellite system resource scheduling model is $\mathrm{PF}^{[4]}$,and the physical layer and link layer mapping methods are $\mathrm{EESM}^{[5]}$.

Table 1 Link parameter for LEO satellite system

\begin{tabular}{cc}
\hline link index & data \\
\hline Spaceborne equipment EIRP (dBW) & 38.02 \\
The average EIRO of Mobile terminal(dBW) & -4.7 \\
Spaceborne receiver power detection threshold & -156 \\
(dBW) & \\
The attenuation of atmospheric, rain, etc. $(\mathrm{dB})$ & 0.7 \\
mobile terminal receiving gain (dB) & 2.6 \\
Satellite receiving antenna gain (dB) & 3.6 \\
bandwidth(MHz) & 10 \\
Spaceborne equipment noise temperature (dBK) & 22.8 \\
Mobile terminal noise temperature (dBK) & 26.6 \\
\hline
\end{tabular}

Table 2 is for a Gaussian white noise channel. When the BLER is not more than 0.1, different CQIs represent different channel characteristics, and user terminals use different modulation methods. The modulation and coding method chosen by AMC is completed by reference [6]. 
Table 2 CQI Correspondence to physical modulation and code pattern

\begin{tabular}{ccccl}
\hline Numble & modulation & bit rate & Mapping factor & SNR \\
\hline 1 & QPSK & 0.0762 & 1.71 & -5.50 \\
2 & QPSK & 0.1172 & 1.32 & -3.75 \\
3 & QPSK & 0.1185 & 1.35 & -2.75 \\
4 & QPSK & 0.3008 & 1.78 & -0.75 \\
5 & QPSK & 0.4385 & 1.77 & 0.75 \\
6 & QPSK & 0.5879 & 1.45 & 2.50 \\
7 & 16QAM & 0.3691 & 4.52 & 6.0 \\
8 & 16QAM & 0.4785 & 5.22 & 6.5 \\
9 & 16QAM & 0.6016 & 4.57 & 8.75 \\
10 & 64QAM & 0.4551 & 4.13 & 13 \\
11 & 64QAM & 0.5537 & 5.07 & 14 \\
12 & 64QAM & 0.6504 & 4.94 & 14.75 \\
13 & 64QAM & 0.7539 & 8.39 & 16 \\
14 & 64QAM & 0.8525 & 15.22 & 18 \\
15 & 64QAM & 0.9258 & 20.91 & 19.25 \\
\hline
\end{tabular}

Figure 3 shows the relationship between the CQI value and the SNR received by the system. From the figure, it can be known that the CQI value can be determined by obtaining the signal to noise ratio of the user signal, and the user terminal can select the appropriate physical layer mapping and coding method for the information transmission through the CQI. Figure 4 shows the performance of the BER block of a system corresponding to different CQI values under the condition of a Gaussian white noise channel.

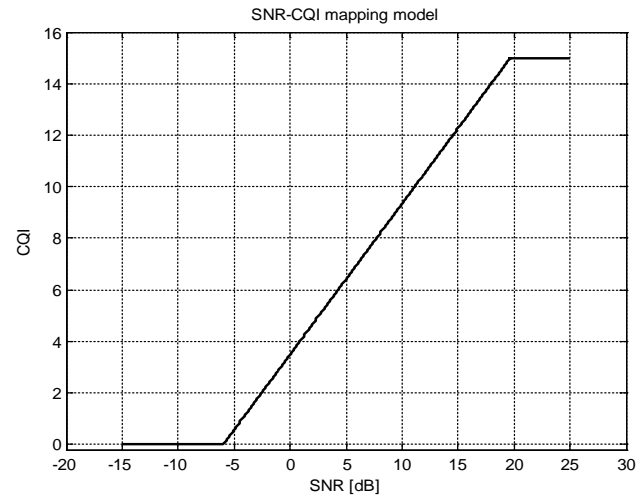

Fig.3 the mapping curve of SNR ratio and CQI

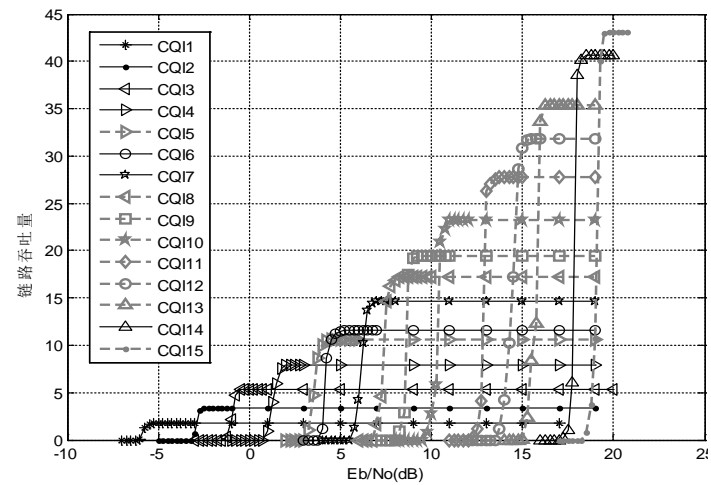

Fig.5 Different CQI throughput under AWGN (under $10 \mathrm{MHz}$ bandwidth)

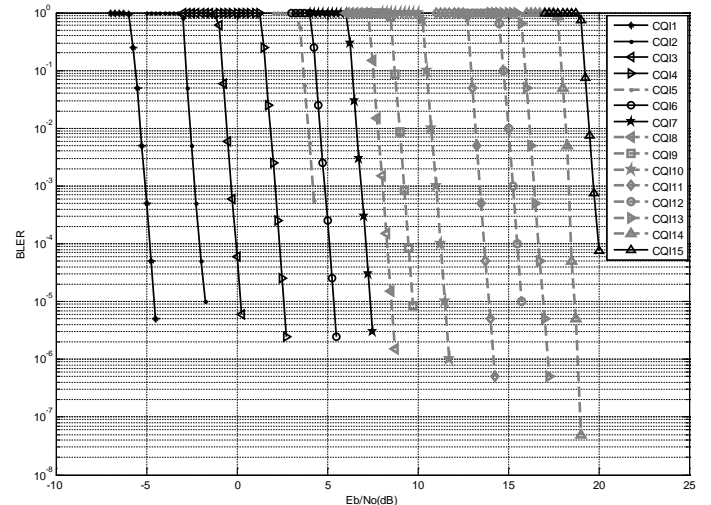

Fig.4 different CQI BER block performance

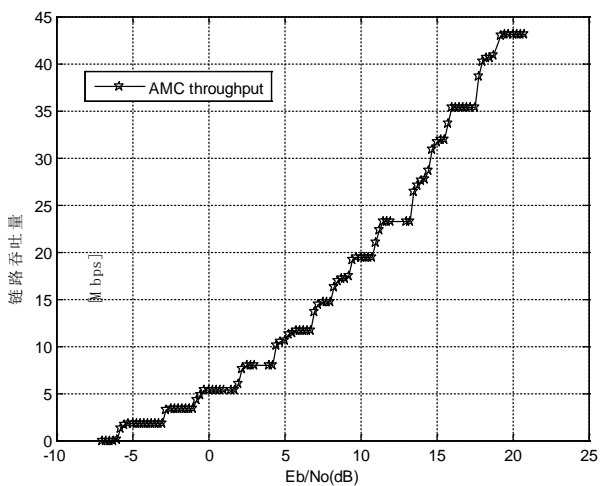

Fig. 6 Adaptive modulation coding throughput under AWGN (10MHz bandwidth)) 


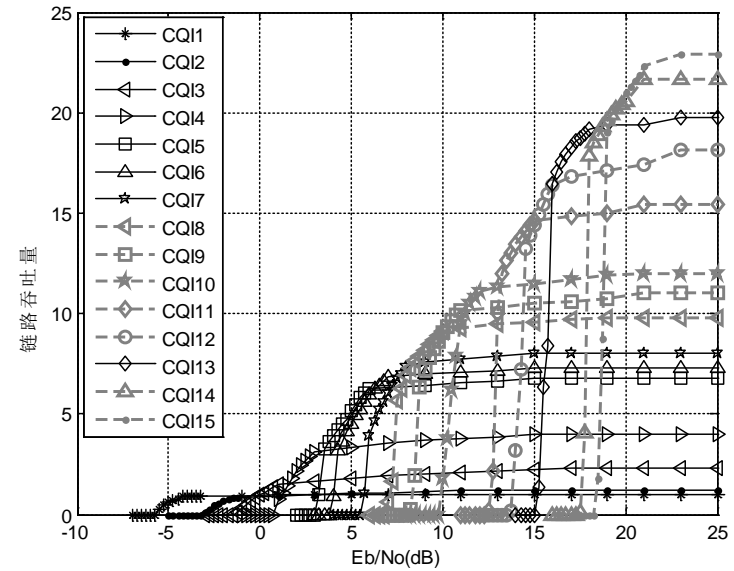

Fig. 7 Different CQI throughputs under 10

$\mathrm{MHz}$ bandwidth in multi-path simulation scenarios for aviation satellites

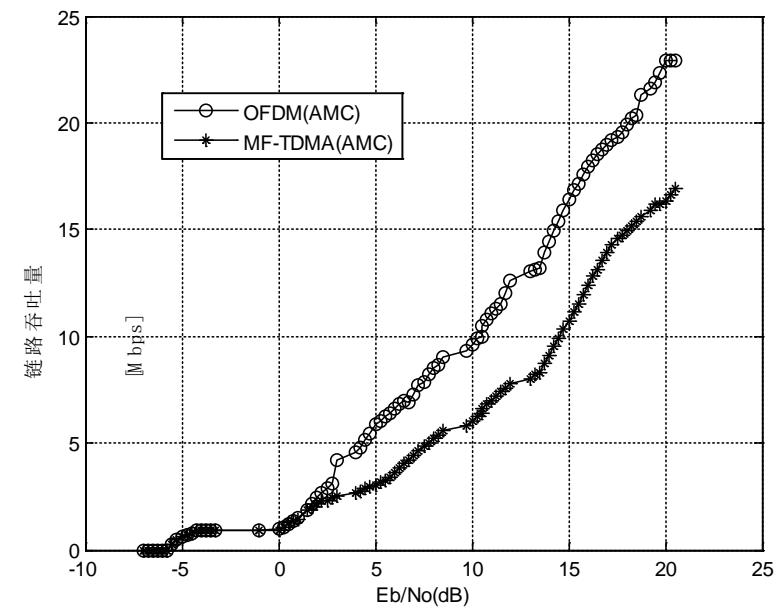

Fig. 8 The comparison of system throughput of two systems in rural simulation scenarios (at $10 \mathrm{MHz}$ bandwidth)

Figure 5 shows the system link throughput performance of different modulation and coding schemes for different CQI values within a $10 \mathrm{MHz}$ signal transmission bandwidth under Gaussian white noise channel conditions.

Figure 6-8 shows the throughput performance of different CQI systems in an aviation broadband satellite link within a $10 \mathrm{MHz}$ signal transmission bandwidth in a rural service scenario. Compared with the AWGN channel condition, the throughput performance of the system link is reduced due to the fading interference of the multipath channel.

In order to analyze the link throughput of the aviation satellite OFDM system, compare with the link throughput of the aviation satellite MF-TDMA transmission system. MF-TDMA is a hybrid multiple access method that combines FDMA and TDMA systems. As the mainstream transmission system adopted by broadband multimedia satellite communication systems at present, MF-TDMA allows user terminals to share a series of carriers with different rates, each carrier is divided into time slots, and resources are flexibly allocated by comprehensive scheduling of time-frequency two-dimensional resources. . In order to simplify the simulation, 20 TDMA symbols are made into subframes, and each 5 subframes constitute one MF-TDMA superframe; the number of MF-TDMA carrier frequency slots is 256, and the time slots are allocated in a uniform manner within one data frame. The satellite receiver completes the user signal detection according to the MMSE criteria. Figure 8 compares the throughput performance of the two transmission systems within a $10 \mathrm{MHz}$ transmission bandwidth under the multipath model. Because OFDM transmission technology adopts orthogonal subcarriers for signal transmission, compared with the MF-TDMA transmission system, it has better anti-channel fading performance and obtains better link throughput performance to some extent.

\section{Conclusions}

This paper analyzes the link AMC throughput of the aviation broadband OFDM satellite mobile communication system. Firstly, it gives an analysis model of the link throughput of the aviation broadband satellite system. Then it introduces the aviation satellite beam coverage model and signaling interaction; High-speed movement, channel fading conditions change, airborne user terminals update CQI and select the appropriate link coding modulation; Finally, the link throughput of MF-TDMA system of aviation broadband satellite communication is compared to prove the link of the OFDM system of the aviation broadband satellite. The superiority of throughput performance. 


\section{Acknowledgments}

This work was financially supported by National Natural Science Foundation of China (No. 61501306) and The Natural Science Foundation of Liaoning Province of China (No. 2015020026).

\section{References}

[1] Tie Zhao, The analysis of physical layer of LTE air interface, J. Telecommunications Technology. 9 (2009):81-83.

[2] Xiao Zhao. The study and simulation in system level of several core techniques about 3GPPLTE, D.Wuhan: Wuhan University of Technology, 2008.

[3] Chao Shun. Implementation of system level simulation platform for the downlink of E3G system and ITS technique’s study, D. Beijing: Beijing University of Posts and Telecommunications, 2009.

[4] Wei Chen, Yin Sun, Yunzhou Li, Realization of System-Level Simulation Platform of LTE Based on Matlab,J. Communications Technology. 43 (2010):170-173.

[5] Haiming Wang, Esa Tuomaala, Effective SINR Approach of Link to System Mapping in OFDM/ Multi - Carrier Mobile Network, J. Sci. Telecommunication Engineering. 1 (2006) 26-30.

[6] 3rd Generation Partnership Project. Physical layer procedures for evolved universal terrestrial radio access (E-UTRA). 3GPP TS 36.213[S]. 3GPP, 2009. 